\title{
Ueber Antipyrin und einige Reactionen desselben.
}

Von Dr. Otto Schwoissingor in Meidelberg.

Die neuere organische Chemie hat seit längerer Zeit sich als ein Hauptziel gestellt die Ergründung der Constitution der Alkaloide und speciell des Chinins, um hieraus Anhaltspunkte für die künstliche Darstellung dieser Körper zu gewinnen. Trotz der ungeheuren Arbeitskraft, welche dieses Gebiet verschlang, waren aber die Resultate verhältnissmässig geringe. Es konnte jedoch constatirt werden, dass die meisten Pflanzenalkaloide den Charakter tertiärer Basen tragen, ferner dass sie zu den Pyridin- und Chinolinkörpern in naher Verwandtschaft stehen und dass besonders der letztere Körper, das Chinolin, $\mathrm{C}^{9} \mathrm{H}^{7} \mathrm{~N}$, wahrscheinlich den Kern dieser wichtigen Pflanzenbasen ausmacht. Auf dieser Annahme beruhten die Versuche der letzten Jahre, Chinolin, Chinolinsalze und Chinolinderivate als Ersatzmittel des Chinins einzuführen. Diese Vorsuche dürfen jedoch alle als ziemlich gescheitert angesehen werden. Neuere Forschungen fülırten dann zu der Ansicht, dass nicht das Chinolin als solches, sondern ein hydrirter Chinolinkern in dem Chinin enthalten sei. Dio Darstellung des Kairins, Oxychinolinmethylhydrür, war ein Versuch in dieser Richtung; trotzdem hat dieser Körper, an den sich anfangs so grosse Hoffnungen knüpften, diese nicht erfüllt.

Das neueste Mittel, welches wegen seiner grossen antipyretischen Wirkung den Namen Antipyrin orhielt, scheint jedoch in der That alle Vorgänger überflügelt zu haben. Das Patent dieses Körpers besitzt Herr Dr. Knorr in Erlangen, die Alleindarstellung hat die chemische Fabrik von Meister, Lucius und Brüning in Höchst. Die ersten Klinischen Versuche mit diesem neuen Körper stellte Herr Prof. Dr. Filehne in Frlangen an, der ausser diesem Körper auch noch eine ganze Reihe anderer Chinolinderivate pharmacologisch prüfte. Nach diesen Untersuchungen (Zeitschrift für klin. Med. Bd. VII H. 6) und nach den seither eingeholten Gutachten oiner grossen Anzall bedeutender deutscher Aerzte bewirkt das Antipyrin bei hochgradigem Fieber eine Temperaturerniedrigung von 2 bis $3^{0}$ ohne unangenehme Nebenerscheinungen. Das Antipyrin wird in Dosen zu 2 und zu 1 g. (für Kinder zu 1 und 0,5 g.) abgetheilt und in Wasser oder in Wein vor jeder Darreichung gelöst. Zur Erzielung einer Temperaturerniedrigung genügen 3 bis $5 \mathrm{~g}$., welche in drei Dosen mit je einer Stunde Intervall $(2+2+1$ resp. $1+1+1)$ zu geben sind. 
Die Fabrik von Meister, Lucius und Brüning bringt jetzt das Mittel in kleinen Blechdosen von $25 \mathrm{~g}$. Inhalt, sowie auch in 0,25 KiloPackung in den Handel. Das Antipyrin wird sich voraussichtlich dauernd im Arzneischatz halten, und es sollen daher im Nachstehenden einige Reactionen, wolche zur Erkennung und Unterscheidung von anderen jetzt gebräuchlichen Antipyreticis dienen, angegeben werden.

Da dio Pharmacopöe ja weder das Kairin noch das Antipyrin kennt, die ständige Pharmacopöo-Commission, welcher hauptsächlich die Aufgabe zufällt, die Prüfungsvorschriften neu auftauchender Mittel zu geben, noch nicht ins Leben getreten ist, trotzdem aber der Apotheker für die Güte und Reinheit auch neuer Mittel verantwortlich bleibt, so dürften die hier folgenden Andeutungen vielleicht Manchem willkommen sein.

Obgleich Herr Dr. Knorr bisher nicht gesagt hat, welcher Körpor aus der ihm patentirten Körpergruppe das Antipyrin ist, so ist jedoch aus den nachstehend beschriebenen Eigenschaften desselben zu schliessen, dass es ein Derivat des Oxymethylchinizins ist und vielleicht dem in den „Ber. der deutschen chem. Gesellschaft" (1884 No. 5 pag. 549) beschriebenen Dimethyloxychinizin sehr nahe steht. Das Oxymethilchinizin entsteht durch Einwirkung von Acetessigaether auf Phenylhydrazin. Das dem Herrn Dr. Knorr gegebene D. R.Patent lautet auf die Darstellung von Oxypyrazolen durch Einwirkung von Acetessigestern, ihren Substitutionsproducten und Homologen auf Hydrazine. Es soll hier zur näheren Orientirung der Biedermann'sche Patentbericht (Ber. der deutsch-chem. Ges. XVII 5, 149) wiedergegeben werden.

„Ein Oxypyrazol ist ein Körper, der durch Condensation von Acetessigester mit einem Hydrazin unter Wasser und Alkoholaustritt entsteht. Acetessigester vereinigt sich mit Phenylhydrazin unter Wasseraustritt zu dem einfachen Condensationsproduct von der Formel

$$
\stackrel{\mathrm{C}^{6} \mathrm{H}^{5}}{\mathrm{H}}>\mathrm{N}-\mathrm{N}==\mathrm{C}\left(\mathrm{CH}^{3}\right) \mathrm{CO}^{2} \mathrm{C}^{2} \mathrm{H}^{5} .
$$

Dieses Product wird beim Vermengen beider Componenten als gelbes Oel erhalten, welches beim Erwärmen unter Alkoholaustritt in Methylphenyloxypyrazol, einen festen Körper ron der Formel $\mathrm{C}^{10} \mathrm{H}^{10} \mathrm{~N}^{2} \mathrm{O}$ übergeht. Man erhitzt den Phenylhydrazinacetessigester auf $100^{\circ}$, bis eine Probe beim Erkalten oder Uebergiessen mit Aether fest wird. Die Reactionsmasse wird noch warm in Aether gegossen 
und die ausgeschiedene Krystallmasse in Aether gewaschen. Methylphenyloxypyrazol giebt sowohl mit Basen als mit Säuren Salze. Sein Schmelzpunkt liegt bei $127^{\circ}$. Beim Frhitzen mit Halogenalkylen auf $100^{\circ}$ entstehen sehr leicht Alkylderivate. Das Methylderivat z. B. krystallisirt aus Aether in perlmutterglänzenden Blättchen vom Schmelzpunkt $116^{\circ}$. Durch Wasser entziehende Mittel \%. B. durch einen Ueberschuss von Phenylhydrazin, entsteht aus dem Methylphenyloxypyrazol beim Erhitzen unter Wasseraustritt ein Anhydridkörper von der Formel $\mathrm{C}^{20} \mathrm{H}^{18} \mathrm{~N}^{4} \mathrm{O}$. In gleicher Weise wie Phenylhydrazin reagiren unter Wasser und Alkoholaustritt die aus Toluidin etc. erhaltenen Hydrazine auf Acetessigaether und seine Substitutionsproducte."

Das schon erwähnte Dimethyloxychinizin wird gewonnen durch Erhitzen eines Gemenges gleicher Theile Methyloxychinizin, Jodmethyl und Methylalkohol im Rohr auf $100^{\circ}$. Die Reactionsmasse wird durch Kochen mit schwefliger Säure entfärbt, der Alkohol abdestillirt und durch Zusatz von cone. Natronlauge das Dimethyloxychinizin als schweres Oel abgeschieden.

Nach dem von der Firma Meister, Isucius und Brüning versandten Circular ist das Antipyrin ein sauerstoffhaltiges Alkaloid, welches als freie Base in den Handel gebracht wird; es bildet mit Säuren Salze, aus denen Alkali die Base wieder in Freiheit setzt. Die empfindlichste Reaction auf Antipyrin besteht in seinem Verhalten gegen oxydirende Agentien. Eisenhaltiger Staub erzeugt in dem reinen Krvstallpulver rothe Flecken; beim längeren Stehen am Lichte wird es ebenfalls etwas roth gefärbt.

Das Antipyrin, wie es heute in den Handel kommt, bildet ein voluminöses, krystallinisches Pulver von röthlich grauer bis weisser Farbe, unter dem Mikroskop zeigt es kleine Blättchen und unvollkommene Säulen. Der Geschmack ist anfangs etwas scharf bitter; jedoch bei weitem nicht so stark wie beim Chinin, und sehr wenig lange anhaltend. Das Antipyrin kann daher leicht in Wein, aber auch ganz ohne Corrigens genommen werden. Es ist in 50 Theilen Aether löslich und krystallisirt bei schnellerer Verdunstung in kleinen glänzenden Blättchen, bei langsamerer in kleinen schiefen rhombischon Säulen. Der Schmelzpunkt liegt bei $113^{\circ}$. In Wasser ist das Antipyrin ausserordentlich löslich; bei $15^{\circ}$ werden 10 Theile des Salzes von 6 Theilen Wasser gelöst, bei etwas höherer Temperatur steigert sich die Löslichkeit sehr; es ist jedoch nicht möglich, eine kalte 
Lösung von 10 Theilen Antipyrin in 5 Theilen Wasser, wie dieselbe von einer Sejte zur subcutanen Injection vorgeschlagen war, herzustellen. Bereitet man eine solche Lösung warm, so krystallisirt nach einiger Zeit, im unglücklichsten Falle während des Gebrauchs, ein Theil des Salzes heraus. Bei höheren Temperaturen steigt jedoch, wie schon bemerkt, die Iöslichkeit ausserordentlich und es kann durch geringe Mengen anhängenden Wassers die Krystallisation verhindert werden. Aus der wässerigen Lösung kann man das Antipyrin in schönen, zollangen, schiefen rhombischen Säulen gewinnen. Alkohol und Chloroform lösen die Krystalle mit Leichtigkeit. Beim Erhitzen schmilzt der Körper zunächst, färbt sich darauf roth, dann braun und lässt einen braunen, brenzlich wie Bernsteinoel riechenden Rückstand, der sich in Chloroform mit rother Farbe und auch in Alkohol löst, dagegen wonig löslich ist in Aether und unlöslich in Benzin, Terpentinöl und Kalilauge. Salzsäure ist ohne Einfluss auf den Körper, Salpetersäure (1,185 spec. G.) in der Kälte ebenfalls. Beim Erwärmen tritt aber plötzlich Rothfärbung ein und bei weiterem Erhitzen findet Abscheidung eines purpurrothen Oeles und eines braunen harzartigen Körpers statt.

Die meisten Reagentien der Alkaloide geben mit Antipyrin Niederschläge, T annin giebt in sehr verdünnten Lösungen einen weissen Niederschlag, in stärkeren Lösungen scheidet es eine schwach gelbe, harzige Masse $a b$, welche in Alcohol löslich ist. Jod-Jodkalium giebt einen rothgelben, in der Wärme löslichen Niederschlag und mit Jodquecksilber-Jodkalium entstcht eine gelbe Färbung; nach einiger Zeit werden kleine gelbe Krystalle abgeschieden. Platinchlorid scheidet aus der Lösung ölig gelbe Tropfen $a b$, die sich beim Erhitzen lösen, bcim Erkalten aber sofort wieder ausscheiden; in verdünnten Lösungen tritt diese Reaction nicht auf. Zinnchlorür erzeugt einen dicken, flockigen, weissen Niederschlag; Quecksilberchlorid nur in concentrirten Lösungen einen weissen, beim Erwärmen löslichen, salpetersaures Quecksilberoxyd dagegen auch in verdünnter Lösung einen Niederschlag. Concentrirte Sch wefelsäure ist nicht von besonderer Einwirkung, nur eine schwache Gelbfärbung wird beobachtet. Mit Pikrinsäure entsteht in concentrirten Lösungen eine gelbe Fällung, welche beim Erwärmen löslich ist. Chlorkalklösung giebt in der schwach mit Salzsäure angesäuerten Lösung eine Trübung, in concentrirteren Lösungen einen gelblich weissen Niederschlag. Kohlensaures Kali scheidet 
ein schwach gelbliches $\mathrm{Oel} a \mathrm{~b}$, wclches ohne Reaction auf Chlorkalklösung ist.

Die wichtigsten Reagention für die Erkennung des Antipyrins und für die Unterscheidung von anderen Körpern sind jedoch Eisenchloridlösung, salpotrige Säure und rauchende Salpetersäure. Mit Eisenchlorid tritt eine rothbraune Färbung auf welche derjenigen am nächsten kommt, die auch Sulfocyankalium in etwas concentrirterer Ijösung giebt. Zur Vergleichung wurden Versuche angestellt mit folgenden Körpern: Carbolsäure, Salicylsäure, Resorcin, Kairin, Antipyrin, Chinin. Man kann die Reaction entweder im Reagensglas oder auf einem weissen Porzellanteller vornehmen; im ersteren Falle lässt man zu 1 bis $2 \mathrm{cc}$. der verdünnten Lösung $(1: 1000)$ des betreffenden Stoffes einen Tropfen Eisenchlorid fallen, im letzteren Falle bringt man zu einigen Körnchen der Substanz oder zu einem Tropfon der Lösung vermittelst eines Glasstabes oder eines Capillarröhrchens einen Tropfen des Reagens.

Setzt man zu 2 cc. einer verdünnten Antipyrinlösung einen Tropfen Eisenchlorid, so entsteht sofort eine intensiv rothbraune Färbung, wolche auf Zusatz von einem Tropfen concentrirter Schwefelsäure verschwindet.

Nachstehend sind die Reactionen der verschiedenen Körper in einer Tabelle zusammengestellt.

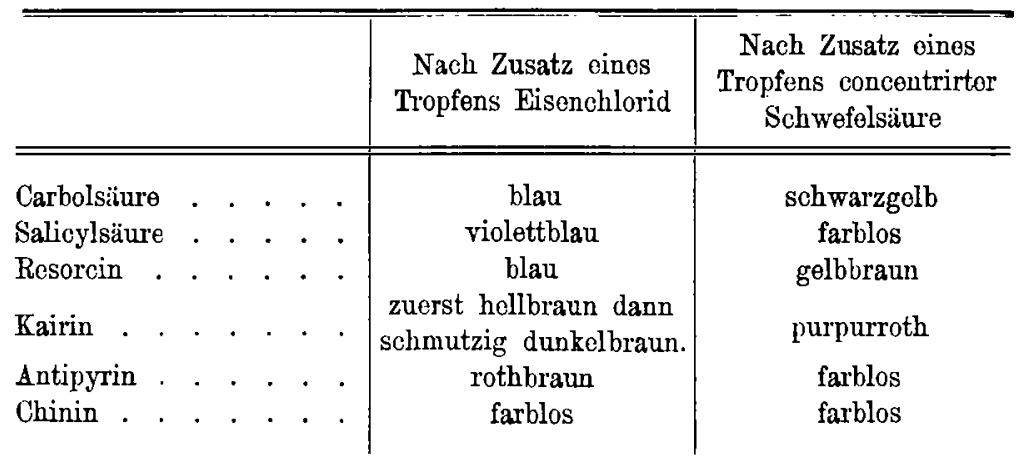

Es genügt also schon diese Reaction, um Kairin und Antipyrin von einander zu unterscheiden; man kann aher auch die Einwirkung einer Chlorkalklösung auf die beiden Stoffe zur Unterscheidung benutzen.

Setzt man zu der neutralen Lösung des Kairins einige Tropfen der Chlorkalklösung, so entsteht eine rothe, bald in schmutzig braun- 
roth ïbergehende Farbe, während bei derselben Reaction Antipyrin vollkommen farblos bleibt. Giebt man jetzt einen Tropfen Salzsäure hinzu, so wird die Lösung des Kairins klar gelb, während das Antipyrin einen weissgelben Niederschlag fallen lässt. Ein ferneres Unterscheidungsmittel der beiden Körper ist das salpetersaure Quecksilberoxyd, welches, wie bereits oben erwähnt, mit Antipyrin einen weissen Niederschlag giebt, während mit Kairin eine orangegelbe Farbe, darauf ein schmutzig brauner Niederschlag. eintritt.

Das am meisten charakteristische Reagens, welches Antipyrin von allen anderen jetzt im Handel befindlichen Körpern, und besonders von den andern Antipyreticis unterscheiden lässt, ist salpetrigre Säure. Diese erzeugt in verdünnten Lösungen eine schöne grüne Färbung. Nun ist die salpetrige Säure zwar ein sehr zersetzliches und in pharmaceutischen Laboratorien selten vorhandenes Reagens, man kann sich aber ein für den vorliegenden Fall sehr brauchbares Reagens durch folgendes Verfahren herstellon. Man giebt in den Reagircylinder einige Körnchen arseniger Säure, übergiesst sie mit 2 bis 3 Tropfon rauchender Salpetersäure und erhitzt, bis eine klare Lösung erfolgt ist; setzt man jetzt einige Tropfen Wasser hinzu und lässt abkühlen, so ist das Reagens für den Gebrauch fertig. Man kann es sich entweder für jeden Versuch frisch bereiten oder für eine kleinere Anzahl von Versuchen vorräthig machen. Im letzteren Falle benutzt man zur Darstellung ein Kölbchen.

Zu der nach obigem Verfahren hergestellten Lösung der salpetrigen Säure giebt man einen Tropfen Antipyrinlösung (1:20), und es tritt sofort die oben orwähnte Grünfärbung auf. Will man aber die Herstellung der salpetrigen Säure ganz umgehen, so genügt zur Ausführung der Reaction die rauchende Salpetersäure; auch mit dieser allein tritt jene charakteristische Grünfärbung in genügender Schärfe auf. Man verfährt folgendermassen.

$\mathrm{Zu}$ einem ce. verdünuter Antipyrinlösung ( $1: 1000)$ setzt man einen Tropfen rauchender Salpetersäure, es entsteht die crwähnte grüne Fürbung, welche sich in der Kälte mehrere Tage erhält. Erhitzt man jetzt die Flüssigkeit und setzt dann einen weiteren Tropfen rauchender Salpotersäure hinzu, so färbt sich die Lösung zuerst hellroth, dann blutroth und scheidet bei weiterem Erhitzen ein purpurnes Oel ab, das sich in Chloroform, nicht aber in Schwefelkohlenstoff und Benzin löst. 
Wonn man zu der concentrirten Lösung des Antipyrins in der Kälte salpetrige Säure oder wenig rauchende Salpetersäure setzt, so scheiden sich in der Lösung kleine grüne Krystalle ab; man muss jedoch mit dem Zusatz der rauchenden Salpetersäure sehr vorsichtig sein, sonst tritt sofort Rothfärbung ein.

Der Vollständigkeit halber sollen auch hier die Reactionen folgen, welche zwecks Vergleichung mit anderen Körpern angestellt wurden:

Auf Zusatz von rauchender Salpetersäure wird

Carbolsäure: schmutzig braun-violett.

Salicylsäure: schwach gelbbraun.

Resorcin: in der Kälte dunkelroth.

Kairin: in der Kälte orangeroth.

Chinin: bleibt farblos.

Antipyrin unterscheidet sich daher ganz scharf von den anderen Körpern und zwar

1) durch die Braunfärbung mit Eisenchlorid und das Verschwinden dieser Farbe mit concentrirtor Schwefelsäure.

2) durch die Zersetzung mit rauchender Salpetersäure. (Grünfärbung, darauf Rothfärbung und Abscheidung eines rothen öligen Körpers.

3) durch die Grünfärbung mit salpetriger Säure.

Besonders die letzte dieser Reactionen dürfte am besten bei einer Identitätsprüfung verwendbar sein.

Da bei entzündlichen Affectionen, bei welchen das Antipyrin zur Verwendung kommt, auch häufig Calomel gegeben wird, so soll an dieser Stelle noch darauf hingewiesen werden, dass diese beiden Körper nicht ohne Einwirkung auf einander bleiben. Uebergiesst man das Quecksilberchlorür mit einer zehnprocentigen Lösung des Antipyrins, so tritt sofort schon in der Kälte eine Graufärbung ein; diese verschwindet nach einigen Stunden und im Filtrat ist nun deutlich Quecksilber nachweisbar. Aber auch hier verhalten sich concentrirte und verdünnte Lösungen verschieden. Schüttet man z. B. zu einem Gramm Calomel ein Gramm Antipyrin und setzt jetzt allmählich zehn Gramm Wasser hinzu, so entsteht zuerst ein grauweisser voluminöser Niederschlag, und das Filtrat giebt mit Schwefelwasserstoff sofort einen schwarzen Niederschlag, der sich als Schwefelquecksilber erweist. Löst man dagegen ein Gramm Antipyrin in 
zehn Gramm Wasser und setzt jetzt ein Gramm Calomel hinzu, so entsteht der erwähnte voluminöse Niederschlag nicht, und das Filtrat giebt anfangs mit Schwefelwasserstoff nur eine schwache Trübung; erst nach längerer Einwirkung steigert sich der Quecksilbergchalt.

Um nun zu erfahren, ob das Quecksilber im Filtrat als Chlorid enthalten sei, wurde mit Jodkaliumlösung geprüft. Der entstehende weissgelbe Niederschlag verhielt sich jedoch nicht so, wie die Fällung aus einer zur Vergleichung in ähnlicher Stärke hergestellten Sublimatlösung, denn während der letztere nach einiger Zeit in Roth überging und sich zu Boden setzte, behielt der erstere Stunden, ja Tage lang seine weissgelbe Farbe. Genau so wie die von dem Calomel abfiltrirte Lösung verhielt sich auch eine Lösung des Antipyrins, welche mit Sublimat versetzt war. Setzt man zu einer starken Antipyrinlösung ( 1 in 10) Quecksilberchlorid, so entsteht zuerst ein weisser Niederschlag, der sich jedoch in überschüssigem Antipyrin löst. In dieser Lösung wird nun, sobald nicht Quecksil. berchlorid im Ueberschuss ist, durch Jodkalium kein rother, sondern ein weissgelber Niederschlag erzeugt, der sich ebenso verhält, wie der bei der Einwirkung auf Calomel in der Antipyrinlösung erhaltene.

Hieraus scheint hervorzugehen, dass das bei der Zersetzung des Quecksilberchlorürs anfangs gebildete Chlorid nicht als solches, sondern in Form einer organischen Verbindung im Filtrat vorhanden ist.

Durch die Freundlichkeit der Herren Assistenten an der hiesigen medicinischen Klinik, welche mir Material in genügender Weise zur Verfügung stellten, war ich in den Stand gesetzt, auch einige Versuche über den Nachweis des Antipyrins im Harn zu machen. Dieselben wurden, wio sämmtliche anderen hier beschriebenen Versuche in der Apotheke des akademischen Krankenhauses ausgefübrt. Als Reagens wurde das Eisenchlorid benutzt. Setzt man zu $1 \mathrm{cc}$. des betreffenden Harns einen Tropfen Eisenchlorid, so entsteht zunächst eine Fällung der Phosphate; nach Zusatz eines weiteren Tropfens Eisenchlorid und einigem Schütteln oder Erwärmen lösen sich diese und der Harn färbt sich dunkel rothbraun. Das Reagens ist zwar nicht besonders scharf und charakteristisch, jedoch erwiesen sich die übrigen für die reinen lıösungen des Antipyrins verwendbaren Reagentien als unbrauchbar und muss daher einstweilen das Eisenchlorid verwendet werden. Bei zwei Versuchen, welche ich an mir selbst anstellte, erschien nach dem Einnehmen von einem Gramm Anti- 
pyrin die Reaction nach kaum einer Stunde im Harn. Dieselbe war in beiden Fällen 12 Stunden bis 18 Stunden deutlich zu rerfolgen, sio verschwand erst nach 24 Stunden. Der Harn reagirte stets sauer, seine Farbe unterliegt keiner besonders starken Veränderung, nur etwas dunkler war derselbe in manchen Fällen getärbt. Bei einzelnen Personen, welche mehrere Tage hindurch 4 bis 6 Gramm Antipyrin genommen hatten, hielt die Eisenchlorid-Reaction im Harn noch 48 Stunden an, nachdem die Darreichung ausgesetzt war, bei anderen verschwand die Reaction schon nach 24. Stunden. Der Harn eines Mannes, welcher mehrere Wochen hindurch täglich 3 Gramm Antipyrin genommen hatte, zeigte die Eisenreaction noch 4 Tage nach dem Aussetzen des Mittels. Da Aethyldiacetessigsäure eine ebensolche Färbung mit Eisenchlorid giebt, wie Antipyrin, so wurde ein deutlich mit Eisenchlorid reagirender Harn der Destillation unterworfen. Es konnte jedoch auch bei längerem Destilliren ein flüchtiger auf Eisenchlorid reagirender Körper nicht abgetrennt werden.

In Urinen, welche nur kleine Spuren Antipyrin enthalten und stark braun gefärbt sind, ist das Antipyrin nicht unmittelbar durch Eisenchlorid nachzuweisen. In solchen Fällen wurde der Harn zunächst auf ein geringes Volumen abgedampft, durch Digeriren mit Thierkohle entfärbt und mit dem Filtrat die Reactionen angestellt. Wurde der zur Syrupdicke eingedampfte Harn nach einander mit absolutem Alkohol und mit Aether ausgezogen, so erschien die Eisenreaction nur in dem Alkoholauszug, während sie im Rückstand sowohl, wie im aetherischen Auszug nicht mehr auftrat. Es gelang aber nicht ausser mit der Eisenchlorid-Reaction einen bestimmten Nachweis des Antipyrins zu führen.

Es wurde daher, um den Körper möglichst isolirt wieder zu gewinnen, folgender Weg eingeschlagen. Der Harn wurde eingedampft, mit Thierkohle entfürbt und filtrirt. Das auf ein geringes Volumen gebrachte Extrat wurde mit dem mehrfachen Volumen absoluten Alkohols gemischt und von der grossen Menge ausgeschiedener Salze wieder abfiltrirt. Nach dem Eindampfen des Filtrats wurde zum Auskrystallisiren des Harnstoffs bei Seite gestellt. Die Mutterlauge wurde abgegossen und nach nochmaligem Eindampfen und Auskrystallisiren zur Ausführung der Reactionen verwendet.

In dem sehr dunkel gefärbten Harn eines Mannes, welcher nur eine Gabe von 2 Gramin Antipyrin erhalten hatte, gelang es auf diese Weise zwar die Eisenchloridreaction $z$ erzeugen; die Abschei- 
dung des rothen in Chloroform löslichen Oeles mit Salpetersäure trat dagegen nicht deutlich hervor und sollen noch weitere Versuche hierüber sowie über die Wiedergewinnung des Körpers selbst angestellt werden.

\section{Mittheilungen aus dem pharmaceutischen Institute zu Strassburg i. Els.}

\section{Ueber die quantitative Bestimmung der China- alkaloïde.}

Von Y. Shimoyama aus Japan.

I. Bestimmung des Gesammtalkaloïd-Gehaltes der Chinarinden.

Obgleich es eine grosse Anzahl von Methoden zur quantitativen Bestimmung der Alkaloïde der Chinarinden giebt, so lässt sich schon bei einer Anzahl derselben voraussehen, dass sie ihren Zweck nicht erfüllen können, und für andere fehlen die Beweise dafür, dass eine vollständige Gewinnung der Alkaloïde wirklich durch sie erreicht wird. Ich habe deshalb im Verlauf des vergangenen Semesters alle Methoden geprüft und bin dabei schliesslich zu einer solchen gelangt, welche der Veröffentlichung werth erscheint. Ich will in dem folgenden einen kurzen Ueberblick über Versuche mittheilen, welche ich im Laboratorium des Herrn Professor Flückiger auf dessen Veranlassung hin ausführte.

Wie gesagt, erschien eine ganze Reihe von Methoden bei einiger Ueberlegung oder nacb kurzen Versuchen als unbrauchbar. Hierher gehört zuerst das Verfahren von Rabourdin (Fresenius Zeitschrift für analytische Chemie 4, p. 288), ferner dasjenige von Hager (Commentar Pharmacopoea Germanica $\Pi$, p. 118), welches schon II. Meyer prüfte (Archiv der Pharmacie 1882, p. 817), die Methodo von Carles (Journal de Pharmacie et de Chimie 1870, p. 81), zuletzt die Methode von Gunning (Fresenius Zeitschrift für analytische Chemie 9, p. 498), mittelst welches schon Hager und H. Meyer viel zu hohe Zahlen und unreine Producte erhalten hatten. Prunier's Methode (Journal de Pharmacie et de Chemie, 4. Série, Tome 29, p. 135) wäre die erste, welche vielleicht einer genauen Prüfung würdig gewesen wäre, doch ist weder die Extraction mit woingeisthaltigem Chloroform nach Prunier's Vorschrift, noch die Fällung der ausgezo- 